\title{
Entanglement Routers Using Macroscopic Singlets
}

\author{
Abolfazl Bayat, ${ }^{1}$ Sougato Bose, ${ }^{1}$ and Pasquale Sodano ${ }^{2}$ \\ ${ }^{I}$ Department of Physics and Astronomy, University College London, Gower St., London WC1E 6BT, United Kingdom \\ ${ }^{2}$ Department of Physics, University of Perugia, and INFN, \\ Sezione di Perugia, Via A. Pascoli, 06123, Perugia, Italy
}

(Dated: December 6, 2018)

\begin{abstract}
We propose a mechanism where high entanglement between very distant boundary spins is generated by suddenly connecting two long Kondo spin chains. We show that this procedure provides an efficient way to route entanglement between multiple distant sites. We observe that the key features of the entanglement dynamics of the composite spin chain are remarkably well described using a simple model of two singlets, each formed by two spins. The proposed entanglement routing mechanism is a footprint of the emergence of a Kondo cloud in a Kondo system and can be engineered and observed in varied physical settings.
\end{abstract}

Introduction:- A high entanglement between two well separated qubits is the central resource for quantum communication tasks. Entanglement between arbitrary pairs of distant qubits in a multi-qubit network enables the linking of several quantum registers to a single larger computer. It also facilitates the preparation multi-particle entangled states [1], for measurement based quantum computation. One could ask whether many-body systems can serve as mediums for entanglement between arbitrary distant qubits in a multi-site network. Though this is the most important question from an "applied" perspective, the thriving field of entanglement in many-body systems [2] remains focussed on the entanglement of blocks and proximal spins. In fact, long range entanglement between individual spins is notoriously uncommon [3]. There are proposals exploiting weak couplings of distant spins to a spin chain [4, 5], but these have limited thermal stability or a very long time-scale of entanglement generation. Alternatively, a global quench [6] or specific time dependent couplings [7] may generate entanglement, though this decays with the system size. Finally, there is a proposal [8] for distance independent entanglement through a local quench which, however, lacks the versatility of routing entanglement between multiple sites. A few quantum routers have been recently proposed [9], but harnessing a canonical many-body phenomenon for routing still is an open question.

Kondo systems [10-12] are very distinctive in the context of entanglement for at least two reasons. Despite being "gapless", they support the emergence of a length scale $\xi$ - the so called Kondo screening length [10, 11]- which can be tuned by varying only one parameter [10] and reflects in the entanglement [8], making it markedly different from other conventional gapless models. Furthermore, in Kondo systems, the impurity spin is maximally entangled [12] with a block of spins whose spatial extent may be varied at will by tuning $\xi$.

In this letter, we propose a dynamical mechanism by which long range distance independent entanglement may be generated by the switch on of a single coupling suddenly connecting two macroscopic singlets. We show that this mechanism provides an efficient way to route entanglement between various distant parties. By a macroscopic singlet we mean an arbitrarily long spin chain which has been engineered to behave as a Kondo system of pertinent $\xi$ and thereby as a two spin singlet.
Indeed we show that, the key features of our mechanism for the two macroscopic singlets, are remarkably well described by a four spin system made of two singlets.

Simple example:- Let us first consider two spin singlets each formed by only two spins interacting with a Heisenberg interaction of strength $J_{1}^{\prime}$ and $J_{2}^{\prime}$, respectively. The ground state of the composite system is then given by $|g s\rangle=$ $\left|\psi^{-}\right\rangle \otimes\left|\psi^{-}\right\rangle$with $\left|\psi^{-}\right\rangle=(|01\rangle-|10\rangle) / \sqrt{2}$. In this simple setting, one may generate high entanglement between the boundary spins, 1 and 4 by merely turning on an interaction $J_{m}$ between the spins 2 and 3. After quenching, the evolution of the system is ruled by the Hamiltonian $H=$ $J_{1}^{\prime} \vec{\sigma}_{1} \cdot \vec{\sigma}_{2}+J_{2}^{\prime} \vec{\sigma}_{3} \cdot \vec{\sigma}_{4}+J_{m} \vec{\sigma}_{2} \cdot \vec{\sigma}_{3}$ and, since the initial state is a global singlet, time evolution allows for a nonzero overlap only with the singlet subspace of the spectrum of $H$ so that

$$
|\psi(t)\rangle=e^{-i E_{S_{1}} t}\left|S_{1}\right\rangle\left\langle S_{1} \mid g s\right\rangle+e^{-i E_{S_{2}} t}\left|S_{2}\right\rangle\left\langle S_{2} \mid g s\right\rangle,
$$

where, $\left|S_{1}\right\rangle$ and $\left|S_{2}\right\rangle$ are two singlet eigenvectors of $H$ with energy $E_{S_{1}}=-4 J_{m}$ and $E_{S_{2}}=0$ respectively. In order to get maximal entanglement between the boundary spins 1 and 4- after a certain time $t^{*}$ - one has to choose $J_{m}=J_{1}^{\prime}+J_{2}^{\prime}$. Once this condition is satisfied the state of the system at time $t$, up to a global phase, is given by

$$
\begin{array}{r}
|\psi(t)\rangle=\frac{-i \sin \left(2 J_{m} t\right)}{2}(|0011\rangle+|1100\rangle)- \\
\frac{\cos \left(2 J_{m} t\right)}{2}(|1001\rangle+|0110\rangle)+\frac{e^{i 2 J_{m} t}}{2}(|0101\rangle+|1010\rangle) .
\end{array}
$$

Surprisingly, $|\psi(t)\rangle$ depends only on $J_{m}$ and, by tracing out the spins 2 and 3 , one gets the density matrix $\rho_{14}(t)$ of the boundary spins. The entanglement between the spins 1 and 4 may be easily computed using concurrence [13] yielding

$$
E=\max \left\{0, \frac{1-3 \cos \left(4 J_{m} t\right)}{4}\right\} .
$$

Eq. (3) shows that $E$ oscillates with a period of $\frac{\pi}{2 J_{m}}$ and that, at time $t^{*}=\frac{\pi}{4 J_{m}}$, the spins 1 and 4 form a singlet state. In this simple setting one sees that: (i) the entanglement dynamics is determined only by two singlet eigenvectors of $H$; (ii) that maximal entanglement is achieved only when $J_{m}=J_{1}^{\prime}+J_{2}^{\prime}$; (iii) the entanglement dynamics is oscillatory with period $2 t^{*}$, 
(a)

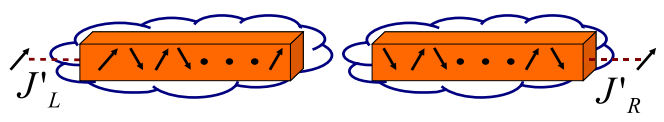

(b)

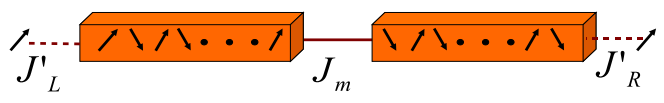

FIG. 1: (Color online) (a) The composite system made of two separate Kondo spin chains initialized in their ground states in the Kondo regime. The extension of the clouds is tuned by $J_{R}^{\prime}$ and $J_{L}^{\prime}$ so that $\xi_{k}=N_{k}-1(k=R, L)$. (b) To induce dynamics, one switches on the interaction between the two chains by the amount $J_{m}$.

\begin{tabular}{|c|c|c|c|c|c|c|c|c|c|}
\hline$N_{k}$ & 4 & 6 & 8 & 10 & 12 & 14 & 16 & 18 & 20 \\
\hline$J_{k}^{\prime}$ & 0.300 & 0.280 & 0.260 & 0.250 & 0.240 & 0.230 & 0.220 & 0.215 & 0.210 \\
\hline \hline$N_{k}$ & 22 & 24 & 26 & 28 & 30 & 32 & 34 & 36 & 38 \\
\hline$J_{k}^{\prime}$ & 0.205 & 0.202 & 0.198 & 0.195 & 0.190 & 0.187 & 0.184 & 0.180 & 0.175 \\
\hline
\end{tabular}

TABLE I: Typical values of $J_{k}^{\prime}$ to generate Kondo clouds of size $\xi_{k}$ as given in Eq. (5).

which is only a function of $J_{m}$ and, thus, does not depend on $J_{1}^{\prime}$ and $J_{2}^{\prime}$ separately.

Generalization to a many-body system:- We now show that the above simple dynamics and the resulting high entanglement between the boundary spins, may be reproduced even with many-body systems- for arbitrary length scales- using pertinent spin chains. For this purpose we consider two Kondo spin chains [10] in the Kondo regime, i.e. two chains of lengths $N_{k}$ described by

$$
\begin{aligned}
H_{k} & =J_{k}^{\prime}\left(J_{1} \vec{\sigma}_{1}^{k} \cdot \vec{\sigma}_{2}^{k}+J_{2} \vec{\sigma}_{1}^{k} \cdot \vec{\sigma}_{3}^{k}\right) \\
& +J_{1} \sum_{i=2}^{N_{k}} \vec{\sigma}_{i}^{k} \cdot \vec{\sigma}_{i+1}^{k}+J_{2} \sum_{i=2}^{N_{k}-2} \vec{\sigma}_{i}^{k} \cdot \vec{\sigma}_{i+2}^{k}, \quad k=R, L(4)
\end{aligned}
$$

where, $J_{1}$ and $J_{2}$ are nearest and next to nearest neighbor couplings, $k=R(k=L)$ labels the right (left) chain, $\vec{\sigma}_{i}^{k}$ is the vector of three Pauli operators at site $i$ for the chain $k$ and $J_{R}^{\prime}$ $\left(J_{L}^{\prime}\right)$ is the impurity coupling of the right (left) hand side.

It is well known that a Kondo spin chain supports a crossover from a gapless Kondo regime for $J_{2}<J_{2}^{c}=$ $0.2412 J_{1}$ to a gapped dimerized regime for $J_{2}>J_{2}^{c}$. In the Kondo regime the Kondo screening length is uniquely determined by the impurity coupling [10,12] and, for large chains, the explicit dependence is given by $\xi_{k}=e^{\alpha / \sqrt{J_{k}^{\prime}}}$, where $\alpha$ is a constant; $\xi_{k}$ sets the size of a block of spins forming a singlet with the impurity [12]. In the following we shall fix the value of $J_{R}^{\prime}$ and $J_{L}^{\prime}$ so that

$$
\xi_{k}=N_{k}-1, \quad k=R, L .
$$

We report in table I the values of the impurity couplings- determined for chains of arbitrary lengths in Ref. [12]- as $N_{k}$ is increased. Eq. (5) allows to build two macroscopic singlets (i.e., extended over a distance $\xi_{k}$ tuned by $J_{k}^{\prime}$ ). The composite spin system is depicted in Fig. 11 a); the two impurities sit at the opposite sides and may be regarded as the boundary spins of the composite system while, due to Eq. (5), the two Kondo clouds are tuned to take over each chain separately. Note that not only is this $J_{k}^{\prime} \sim 1 / \log ^{2} N_{k}$ much stronger than the weak couplings in Refs. [4, 5], but also the chain is gapless, so it cannot lead to perturbative end-to-end effective Hamiltonians.

Initially, the two chains are separated and initialized in their ground states (see Fig. 1a)) and the initial state of the composite chain is given by $|\psi(0)\rangle=\Pi_{k=R, L}\left|G S_{k}\right\rangle$ where $\left|G S_{k}\right\rangle$ is the ground state of the chain $k$. Then, we switch on

$$
H_{I}=J_{m}\left(J_{1} \vec{\sigma}_{N_{L}}^{L} \cdot \vec{\sigma}_{N_{R}}^{R}+J_{2} \vec{\sigma}_{N_{L}-1}^{L} \cdot \vec{\sigma}_{N_{R}}^{R}+J_{2} \vec{\sigma}_{N_{L}}^{L} \cdot \vec{\sigma}_{N_{R}-1}^{R}\right) \text {. }
$$

between the two chains (see Fig. 1(b)). The Hamiltonian of the composite system of length $N=N_{L}+N_{R}$ is given by $H=H_{L}+H_{R}+H_{I}$. Now the ground state evolves according to $|\psi(t)\rangle=e^{-i H t}|\psi(0)\rangle$. From knowing $|\psi(t)\rangle$ one obtains the reduced density matrix of the boundary spins at a generic time $t$ by tracing out all other spins from the state $|\psi(t)\rangle$ and evaluate the concurrence $E\left(t, J_{m}\right)$ between the boundary spins. The dynamics is now not analytically solvable and one has to resort to numerical simulations which, for $N>20$, use the time dependent density matrix renormalization group (tDMRG) introduced in [14] while, for $N<20$, one may use exact diagonalization. For temperatures $T<1 / \xi \sim 2 / N$, i.e. when the two constituent chains are in the Kondo ground state, we find that the evolution of the composite chain well reproduces all the relevant features exhibited by the simple dynamics of a four spin system made out of two singlets.

If the composite system built out of two extended Kondo singlets should reproduce the remarkable features of the simple example discussed above one should expect that, $E\left(t, J_{m}\right)$ oscillates with a period depending only on $J_{m}$ and that maximal entanglement between the boundary spins is reached at the half of the period provided that

$$
J_{m}=\Phi(N)\left(J_{L}^{\prime}+J_{R}^{\prime}\right) .
$$

$\Phi(N)$ accounts for the effects arising due to the extended size of the Kondo singlets. Of course, for our dynamics to make sense at all one has to require that, as $N \rightarrow \infty, J_{m}$ should take a nonzero and finite limiting value $J$ (otherwise, one injects either zero or infinite energy). It is remarkable that this condition alone suffices to determine $\Phi(N)$. Indeed, if $N_{L} \approx N_{R} \rightarrow \infty$, one has

$$
\Phi(N) \sim \frac{J}{\alpha^{2}} \log ^{2}\left(\frac{N}{2}\right),
$$

since, in the Kondo regime, one has that $\xi_{k}=e^{\alpha / \sqrt{J_{k}^{\prime}}}$.

For the time being we will consider only chains for which $N_{L}=N_{R}$ and so that $N=2 N_{L}$. In Fig. 2 a) we plot 

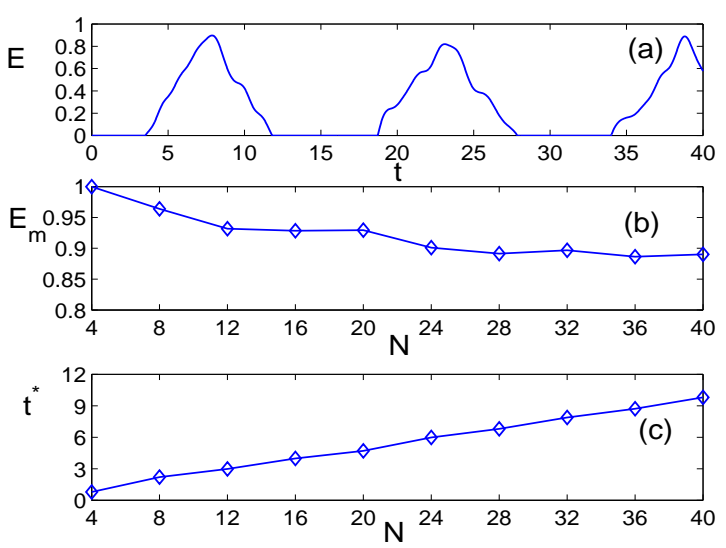

FIG. 2: (Color online) (a) The oscillatory dynamics of entanglement $E$ vs. time $t$ in the Kondo regime $\left(J_{2}=0\right)$ for a composite system of $N=32$ when $N_{L}=N_{R}$. (b) The maximal entanglement $E_{m}$ vs. $N$ at $t=t^{*}$ when $J_{m}$ has its optimal value. (c) $t^{*}$ vs. $N$.

the evolution of the entanglement as a function of time for $J_{m}=0.97 J_{1}$ when $N=32$ in the Kondo regime $\left(J_{2}=0\right)$ of each chain. We see that entanglement dynamics is oscillatory with a period $2 t^{*}$. Restricting only to the first period of oscillations one sees that, there is an optimal value of $J_{m}$ for which, at time $t^{*}$, the entanglement reaches its maximum $E_{m}$. In Fig. 2 b) we plot $E_{m}$ as a function of $N$. Though the entanglement decreases as $N$ increases for short chains, its value remains very high and becomes distance independent for very long chains. It is remarkable that this distance independent value seems to be 0.9 (e.g. for chains of length $N=40$ ) whereas for the only other distance independent case so far [8] it was merely 0.7 . To complete the picture of entanglement evolution in Fig. 2 (c) we plot $t^{*}$ as a function of $N$. We see that the time needed to generate the entanglement between the boundary spins increases linearly with $N$ with a slope that is small enough to allow for fast dynamics. The linear dependence of $t^{*}$ on $N$ implies that, for a system composed of two extended Kondo singlets, $t^{*}$ is related to $J_{m}$ by

$$
t^{*} \sim N \sim \xi_{k} \sim e^{\alpha \sqrt{\frac{2 \Phi(N)}{J_{m}}}} .
$$

In Fig. 3(a) we have plotted the optimal value of $J_{m}$ as a function of $N$. One sees that, as $N$ increases, $J_{m}$ goes to 1 thus, confirming the assumption used in the derivation of $\Phi(N)$ (see Eq. (8)). In Fig. 3(b) we plot $\Phi(N)$ versus $\log ^{2}(N / 2)$. The linearity of the plot provides an independent numerical confirmation of the result obtained in Eq. (8).

Our numerical approach allows to investigate also situations for which $N_{L}$ is different from $N_{R}$. In Fig. 4(a) we plot $E_{m}$ versus $N_{L} / N$ for a composite chain of length $N=32$. Fig. 4 (a) shows that the entanglement is maximal when $N_{L}=N_{R}$ and that decreases sensibly when the sizes of the constituent Kondo chains are very different. In Fig. 4 b) we plot $t^{*}$ as a function of $N_{L} / N$. Again one sees that the optimal time $t^{*}$ is much shorter when $N_{L} \sim N_{R}$. Figs. 4(a) and
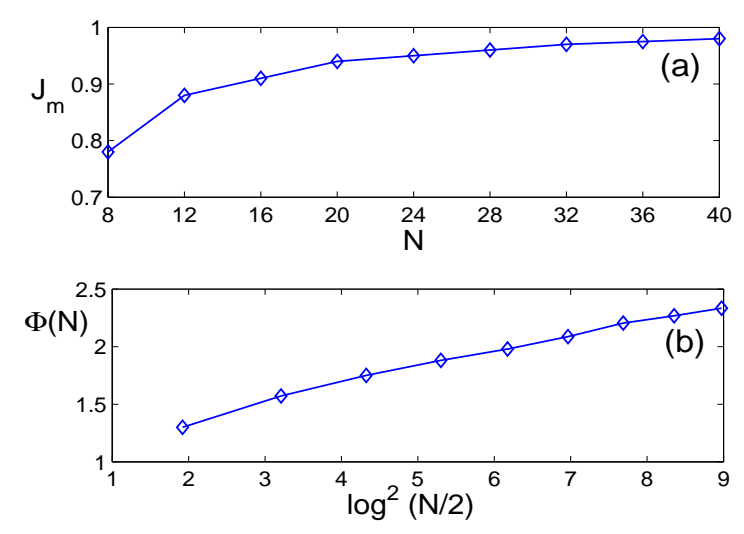

FIG. 3: (Color online) (a) Optimal $J_{m}$ vs. $N$ for $N_{L}=N_{R}$ in the Kondo regime. (b) The asymptotic behavior of $\Phi(N)$ vs. $\log ^{2}\left(\frac{N}{2}\right)$.
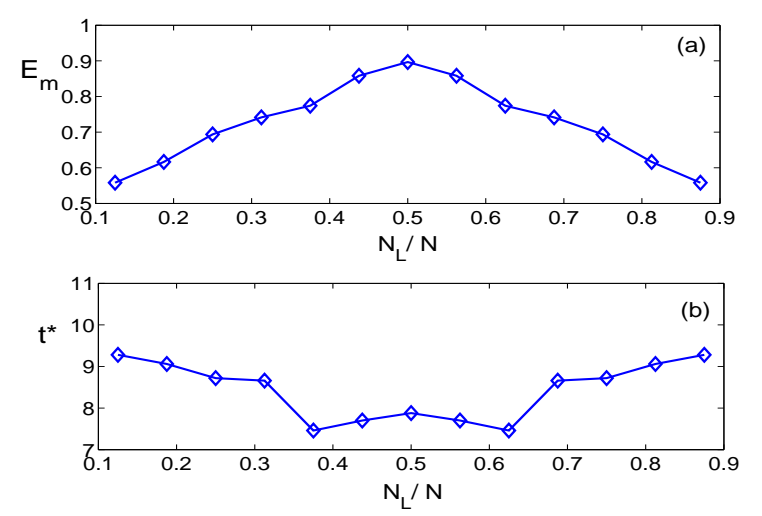

FIG. 4: (Color online) Non-symmetric case where $N=32$ and $N_{L}$ and $N_{R}$ are varied: (a) $E_{m}$ vs. $N_{L} / N$. (b) $t^{*}$ vs. $N_{L} / N$.

(b) lead us to conclude that efficient routing of entanglement is possible only if $N_{L} \sim N_{R}$.

The proposed mechanism for generating high entanglement between the boundary spins of a composite spin system relies heavily on Eq. (5) and, thus, on the fact that, for Kondo chains of arbitrary sizes $N_{L}$ and $N_{R}$, one can always tune the impurity couplings $J_{L}^{\prime}$ and $J_{R}^{\prime}$ so as to make the Kondo cloud comparable with the size of the chains. As a result, entanglement generation between the boundary spins should vanish for $\xi_{k}<N_{k} / 2$ as well as when the constituent Kondo

\begin{tabular}{|c|c|c|c|c|c|c|c|c|c|}
\hline$N$ & 8 & 12 & 16 & 20 & 24 & 28 & 32 & 36 & 40 \\
\hline$E_{m}(\mathrm{~K})$ & 0.964 & 0.932 & 0.928 & 0.929 & 0.901 & 0.891 & 0.897 & 0.886 & 0.891 \\
\hline$E_{m}(\mathrm{D})$ & 0.957 & 0.903 & 0.841 & 0.783 & 0.696 & 0.581 & 0.468 & 0.330 & 0.160 \\
\hline$t^{*}(\mathrm{~K})$ & 2.200 & 2.980 & 3.980 & 4.700 & 5.980 & 6.800 & 7.880 & 8.720 & 9.800 \\
\hline$t^{*}(\mathrm{D})$ & 3.780 & 7.290 & 10.32 & 13.41 & 16.89 & 20.43 & 24.51 & 27.12 & 35.01 \\
\hline
\end{tabular}

TABLE II: Comparison between $E_{m}$ and $t^{*}$ for a Kondo spin chain in the Kondo $\left(J_{2}=0\right)$ and dimer regimes $\left(J_{2}=0.42\right)$. In the table $K$ stands for Kondo and $D$ for dimer. 


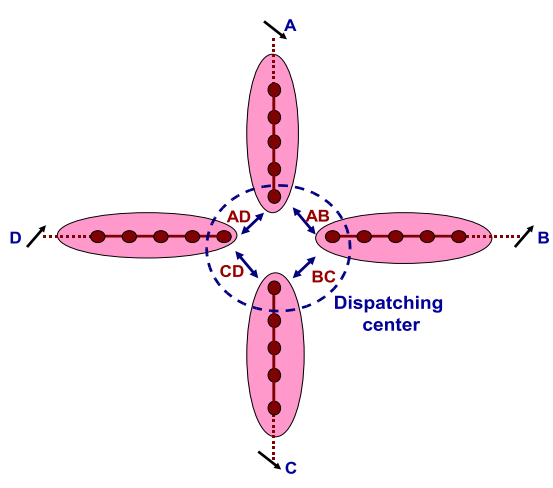

FIG. 5: (Color online) A four-node router in which each user controls one boundary spin. A dispatcher connects two chains to induce dynamics in a channel composed of two spin chains in order to generate entanglement between the boundary spins.

chains are in the dimer regime (i.e., $J_{2}>J_{2}^{c}$ ) where the cloud does not exist at all. We computed numerically $E_{m}$ and $t^{*}$, for a chain composed of two Kondo spin chains in the dimer regime. The results are reported in table II and compared with the results obtained for the same quantities when the two constituent chains are in the Kondo regime and Eq. (5) is satisfied. Table II shows that, as $N$ increases, entanglement $E_{m}$ (optimal time $t^{*}$ ) is very small (large): for instance, for $N=40$, in the dimer regime, $E_{m}=0.16$ and $t^{*}=35.01$ while, in the Kondo regime, $E_{m}=0.89$ and $t^{*}=9.80$.

Entanglement router:- Our analysis allows to engineer an efficient entanglement router dispatching entanglement between very distant qubits. A four-node entanglement router is sketched in Fig. 5 Each node, say $A, B, C$ and $D$, has a boundary spin whose coupling to its adjacent chain is tuned so as to generate a Kondo cloud reaching the dispatch center (Fig. 5). The dispatcher can entangle the spins of two arbitrarily chosen nodes, say $A$ and $B$, by switching on a coupling $J_{m}$ between the chains $A$ and $B$ and, thus, induce the quench dynamics previously analyzed. At $t=t^{*}$, the entanglement may be taken out of the boundary spins by a fast swap to any memory qubits in nodes $A$ and $B$ for building resources for quantum computation. Note that exclusive pairs of nodes, e.g. $(A, B)$ and $(C, D)$, can be connected simultaneously.

Implementations and challenges:- Spin chains with switchable/tunable couplings are realizable [15] with both superconducting qubits and spins in quantum dots. In the former, the effect of a reasonable dephasing of strength $0.005 J_{1}$ [16] for $N=12$ is about $10 \%$. In the latter, a magnetic field in a random direction acts on each spin due to the dot nuclei [17]. Our simulations show that for $N=12$, a very strong magnetic field $\left(\sim 0.05 J_{1}\right)$ [17] decreases the entanglement by $5 \%$.

Conclusions:- We proposed a mechanism for generating high entanglement between distant spins by switching on an appropriate interaction between two Kondo spin chains. In contrast to other recent networking schemes [9] it does not demand control of the intermediate spins or time-varying local fields. Our results hint that a Kondo spin chain satisfying Eq. (5) may be effectively described by an extended singlet formed by two spins since the key features of the entanglement dynamics can be easily understood using a simple model of a pair of two spin singlets. Indeed, in this non-solvable model, the above is the best explanation of the strikingly high entanglement. From table II one sees that in the absence of the Kondo cloud, entanglement is suppressed; thus, the remarkable dynamical behavior of the system is a new clear footprint of the emergence of the Kondo cloud in a Kondo system.

Acknowledgements:- Discussions with I. Affleck, H. Johanneson, N. Laflorencie and E. Sorensen are acknowledged. AB and SB (P.S.) thank(s) the University of Perugia (UCL) for hospitality and partial support. SB and AB acknowledge the EPSRC, and the Royal Society and the Wolfson Foundation.

[1] S. C. Benjamin, B. W. Lovett and J. M. Smith, Laser Photon. Rev. 3, 556 (2009).

[2] L. Amico, et. al., Rev. Mod. Phys. 80, 517 (2008).

[3] A. Osterloh, et. al., Nature 416, 608-610 (2002); T. J. Osborne and M. A. Nielsen, Phys. Rev. A 66, 032110 (2002).

[4] L. Campos Venuti, C. Degli Esposti Boschi and M. Roncaglia, Phys. Rev. Lett. 96, 247206 (2006); L. Campos Venuti et. al., Phys. Rev. A 76, 052328 (2007).

[5] L. Campos Venuti, C. Degli Esposti Boschi and M. Roncaglia, Phys. Rev. Lett. 99, 060401 (2007); M. J. Hartmann, M. E. Reuter, M. B. Plenio, New J. Phys. 8, 94 (2006); Y. Li et. al., Phys. Rev. A 71, 022301 (2005); A. Wojcik et. al., Phys. Rev. A 72, 034303 (2005).

[6] H. Wichterich, S. Bose, Phys. Rev. A 79, 060302(R) (2009); K. Sengupta, D. Sen, Phys. Rev. A 80, 032304 (2009).

[7] F. Galve et. al., Phys. Rev. A 79, 032332 (2009).

[8] P. Sodano, A. Bayat, S. Bose, Phys. Rev. B 81, 100412(R) (2010).

[9] D. Zueco, et.al., Phys. Rev. A 80, 042303 (2009); P. J. Pemberton-Ross and A. Kay, arXiv:1007.2786

[10] E. S. Sorensen, et. al., J. Stat. Mech., P08003 (2007); N. Laflorencie, E. S. Sorensen and I. Affleck, J. Stat. Mech., P02007, (2008);

[11] I. Affleck, Lecture Notes, Les Houches 2008, arXiv:0809.3474

[12] A. Bayat, P. Sodano and S. Bose, Phys. Rev. B 81, 064429 (2010).

[13] W. K. Wootters, Phys. Rev. Lett. 80, 2245 (1998).

[14] S. R. White and A. E. Feiguin, Phys. Rev. Lett. 93, 076401 (2004).

[15] D. Giuliano and P. Sodano, Nucl. Phys. B 811, 395(2009); F. Meier, J. Levy, and D. Loss, Phys. Rev. Lett. 90, 047901 (2003).

[16] J. J. G. Ripoll, et. al., Phys. Rev. B 77, 024522 (2008).

[17] J. M. Taylor, et. al., Phys. Rev. B 76, 035315 (2007). 